\section{Fractionation of Iron in the Solar System}

Harris and Tozer ${ }^{1}$ have recently suggested that mag. netostatic attraction between cosmic iron particles is responsible for the observed variation in iron abundance between the inner and outer planets of the solar system. I should like to point out that a crucial assumption in this model is "the uniform single-domain nature" of iron particles in the size range $10^{-4}-10^{-3} \mathrm{~cm}$. The calculations which follow show that iron particles of only a limited size range can take part in magnetostatic accretion.

At room temperature, $300^{\circ} \mathrm{K}$, iron is superparamagnetic ${ }^{2}$ below the critical size of $125 \AA$. This implies that such particles have no net magnetic momont because of thermal fluctuations. Above that diameter, iron is definitely a single domain but the eritical diameter for the onset of multidomain behaviour is very close, $150 \AA$. As Table 1 shows, at $950^{\circ} \mathrm{K}$, the temperature of accretion considered by Harris and Tozer, the superparamagnetic eritical size is raised to $184 \AA$ and an upper limit of $3300 \AA$ emorges for the multidomain behaviour. This upper limit eomes from the well known fact that a single-domain particle has to be smaller than the domain wall width at the tem. perature being considored. The width of $3300 \AA$ at $950^{\circ} \mathrm{K}$ is dorived from the data given by $\mathrm{Kittel}^{3}$. The sizes considered by Harris and Tozer, $10^{-4}-10^{-3} \mathrm{~cm}$, are far larger than the ones shown in Table 1, and thus will have to be multidomained.

\begin{tabular}{|c|c|c|}
\hline Truperature & $\begin{array}{l}\text { Superparamagnetic } \\
\text { eritical diameter }\end{array}$ & $\begin{array}{l}\text { Single-domain } \\
\text { critical diameter }\end{array}$ \\
\hline $\begin{array}{l}300^{\circ} \mathrm{K} \\
950^{\circ} \mathrm{K}\end{array}$ & $\begin{array}{l}125 \AA \\
184 \AA\end{array}$ & $\begin{array}{r}150 \AA \\
<3300 \AA\end{array}$ \\
\hline
\end{tabular}

Why is it so important for the particles to be singledomained? Because, for a multidomain particle of iron tumbling in a maximum field of $100 \gamma\left(10^{-3}\right.$ oersteds), the net moment would be negligible. The value used by Harris and Tozer is 200 gauss, but the real moment could not be greater than a hundredth of this. Because the product of the moments is responsible for the attractive force, the magnetostatic attraction for $10^{-4} \mathrm{~cm}$ particles could be a ten thousandth of what Harris and Tozer have assumed. The influence of magnetism on the gravitational attraction will indeed be there but it will be too small to cause an offective change in the capture cross-section.

It might be argued that at some time in the past the particles could have gone through an intense magnetic field emanating from the Sun, and this could have saturated the $10^{-4}-10^{-3} \mathrm{em}$ particles. But even so, for a sphere of iron of which the crystalline anisotropy energy at $950^{\circ} \mathrm{K}$ is low (about $10^{4} \mathrm{ergs} / \mathrm{cm}^{3}$ ) and the demagnetizing factor is so large, this saturation moment would be very unstable with time, instantly decreasing to the steady state remanence of about a tenth or a hundredth of one gauss, as discussed before.

My conclusion therefore is that, although the mochanism suggested by Harris and Tozer is ingonious and interesting, it is of little importance for the size of particles thoy have discussed. In order to be effective, the particles have to fall in a vory limited range of sizes as shown in Table 1. It may be possible to postulate that the original particles were indeed in this critical range because of somo vagary of cosmology and that the observed particle sizes are larger because of grain growth on sintering.

I thank Professors R. L. White and A. Cox of Stanford University and Dr P. D. Baba of Ampex Corporation for constructive criticisms.

\section{S. K. BANERJEF*}

Ampex Corporation,

Redwood City, California.

Received, October 23, 1967.

* On leave of ahsence from the School of Physics, University of Newcastle upon Tyne.
${ }^{1}$ Harris, P. G., andi Tozer, D. C., Nature, 215, 1449 (1967).

'Morrish, A. H., in The Physical Principles of Magnetism, 340 (John Wiley and Sons, 1965). ' Kittel, C., in Introduction to Solid Stale Physics, 430 (John Wiley and Sons,
1957).

\section{Banded Flints}

BANDED flints have long been known to occur in small numbers together with ordinary flints, which are the nodules of chalcedonic silica found in the chalk (Upper Cretaceous) of England and elsewhere. The bands may be observed on fractured surfaces and show up as a variation in colour within the normal colour range of unbanded flints. The exterior of the affected nodule may be ridged in a pattern conformable to the banding.

Formerly, authors, such as Woodward ${ }^{1}$ and Sollas ${ }^{2}$. explained the banding as being caused by some kind of infiltration, as would be ascribed nowadays to a natural chromatographic process. Geinitz ${ }^{3}$ suggested rhythmic precipitation and Gebhardt ${ }^{4}$ regarded a particular example as a test case in the recognition of Liesegang's rings as a natural phenomenon. Cayeux ${ }^{5}$ figured a similar specimen and noted that the effect, silex zonaire, involved the normal constituents of flint only, but he was unable to suggest any cause.

That these bands follow the patterns formed in experimental stress analysis does not seem to have been previously noted. Such stress patterns may be preserved by the "freezing" of an elastic component in a medium which is only plastic during loading. Synthetic resins, which provide both components, were discovered in 1935 , and have since been used experimentally ${ }^{6}$. Comparison leaves no doubt that banded flints have been subjected to stresses, probably through collision with other flints.

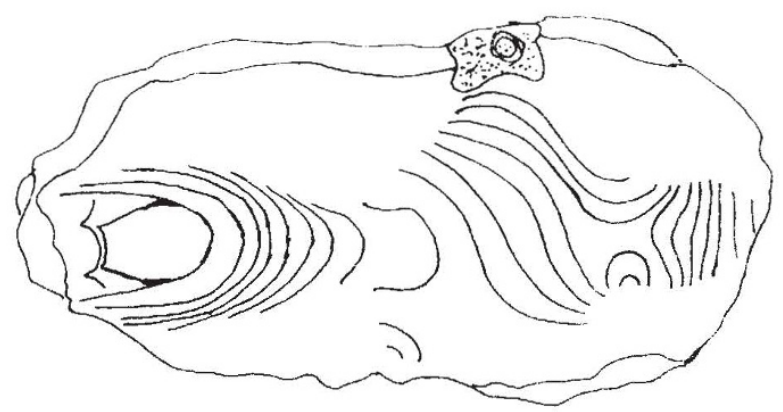

Fig. 1. A sketch of a banded flint. This banded flint is from the collection of Dr W. Hunter, at the University of Glasgow. It is specimen II 117 in the Hunterian Muscum (Geology).

The specimen illustrated in Fig. 1 shows one half of a flint from the collection of Dr William Hunter at the University of Glasgow. The pattern is in black, white, red and yellow according to the amount of compression and subsequent iron-staining. The source of the banded flint is not given but is presumed to be from the oolitic iron-ores of Baden, West Germany, a commercial source for so-called "red Egyptian Jasper", in the eighteenth century. I was privileged to examine Dr Hunter's collection in 1963. A recent article recorded the development of preferred orientation of quartz during recrystallization of flint in conditions of annealing. I suggest here that the naturally banded flints would similarly develop preferred orientation, as a result of the stress "frozen" in the specimen. This is regardless of the demonstration of preferred orientation in a naturally stressed flint, which has not been attempted. The evidence shows that annealing of flint results in preferred orientation which cannot be considered as free from stress, although 